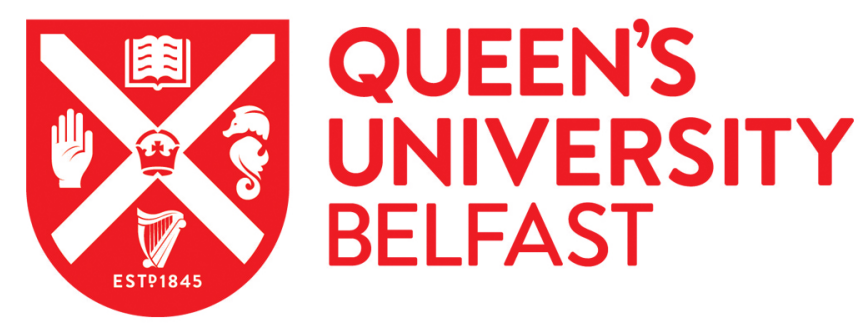

\title{
Channel Characteristics of Dynamic Off-Body Communications at 60 GHz under Line-of-Sight (LOS) and Non-LOS Conditions
}

Yoo, S. K., Cotton, S. L., Chun, Y. J., Scanlon, W., \& Conway, G. A. (2017). Channel Characteristics of Dynamic Off-Body Communications at $60 \mathrm{GHz}$ under Line-of-Sight (LOS) and Non-LOS Conditions. IEEE Antennas and Wireless Propagation Letters. https://doi.org/10.1109/LAWP.2017.2650863

Published in:

IEEE Antennas and Wireless Propagation Letters

Document Version:

Publisher's PDF, also known as Version of record

Queen's University Belfast - Research Portal:

Link to publication record in Queen's University Belfast Research Portal

Publisher rights

(c) 2017 The Authors

This is an open access article published under a Creative Commons Attribution License (https://creativecommons.org/licenses/by/4.0/), which permits unrestricted use, distribution and reproduction in any medium, provided the author and source are cited.

\section{General rights}

Copyright for the publications made accessible via the Queen's University Belfast Research Portal is retained by the author(s) and / or other copyright owners and it is a condition of accessing these publications that users recognise and abide by the legal requirements associated with these rights.

Take down policy

The Research Portal is Queen's institutional repository that provides access to Queen's research output. Every effort has been made to ensure that content in the Research Portal does not infringe any person's rights, or applicable UK laws. If you discover content in the Research Portal that you believe breaches copyright or violates any law, please contact openaccess@qub.ac.uk. 


\title{
Channel Characteristics of Dynamic Off-Body Communications at $60 \mathrm{GHz}$ Under Line-of-Sight (LOS) and Non-LOS Conditions
}

\author{
Seong Ki Yoo, Student Member, IEEE, Simon L. Cotton, Senior Member, IEEE, Young Jin Chun, Member, IEEE, \\ Wiliam G. Scanlon, Senior Member, IEEE, and Gareth A. Conway, Member, IEEE
}

\begin{abstract}
This letter investigates the first-order characteristics of dynamic off-body communications channels at $60 \mathrm{GHz}$. In particular, we have studied signal propagation from a chest-worn millimeter-wave transmitter as an adult male walked toward and then away from a hypothetical base station. The mobile line-ofsight (LOS) and non-LOS (NLOS) channel measurements have been conducted in a diverse range of environments, including a hallway, an open office, an anechoic chamber, and an outdoor car park. In this study, we have decomposed the received signal into its path loss, large-scale, and small-scale fading components. The large-scale fading has been modeled using the gamma distribution, while the Rice and Nakagami- $m$ distributions have been employed to describe the small-scale fading observed in the LOS and NLOS channel conditions, respectively. The results have shown that the estimated path loss exponents for the anechoic chamber and car park environments were greater than those obtained for the hallway and open office environments for both the LOS and NLOS walking scenarios. Across all environments, it was found that the gamma distribution provided an adequate fit to the large-scale fading. Additionally, the Rice and Nakagami- $m$ distributions were found to well describe the small-scale fading for the LOS and NLOS walking scenarios, respectively.
\end{abstract}

Index Terms-Large-scale fading, millimeter-wave (mm-wave) communications, off-body communications, path loss, small-scale fading.

\section{INTRODUCTION}

$\mathbf{R}$ ECENTLY, much interest has been generated toward the use of millimeter-wave (mm-wave) technologies for bodycentric and wearable systems [1]-[6]. Advancements in this area mean that it will soon be feasible to use operating frequencies in the 59-66-GHz range to provide high bandwidth capabilities for these applications. Operating wearable systems within this part of the mm-wave spectrum will be attractive for many reasons, not least due to the small size of antenna that can be used [2], the lower interference, and much greater frequency reuse that

Manuscript received July 25, 2016; revised November 29, 2016; accepted December 28, 2016. Date of publication February 9, 2017; date of current version June 12, 2017. This work was supported in part by the Engineering and Physical Sciences Research Council under Grant Reference EP/L026074/1 and in part by the Department for Employment and Learning Northern Ireland under Grant Reference USI080.

The authors are with the Institute of Electronics, Communications and Information Technology, Queen's University Belfast, Belfast BT3 9DT, U.K. (e-mail: syoo02@qub.ac.uk; s.cotton@ecit.qub.ac.uk; Y.Chun@qub.ac.uk; w. scanlon@qub.ac.uk; g.conway@qub.ac.uk).

Color versions of one or more of the figures in this letter are available online at http://ieeexplore.ieee.org.

Digital Object Identifier 10.1109/LAWP.2017.2650863 can be achieved over smaller areas [5] compared to competing microwave technologies.

The characteristics of the on-body channel at $60 \mathrm{GHz}$ have been studied in [2] and [3]. In [2], it was shown that the choice of antenna polarization is of great importance, and its impact is significantly influenced by the separation distance between the antenna and the body. In [3], the path loss and temporal fading were studied for differing polarizations. The authors found that the received signal often deviated significantly from its fitted path loss model irrespective of polarization. They also adopted a Gaussian distribution to characterize the temporal fading observed in on-body links at $60 \mathrm{GHz}$ and subsequently derived a model for the standard deviation. Similarly, an off-body propagation model at $60 \mathrm{GHz}$ was developed using theory in [6] and validated using empirical measurements. In [6], the path gain was considered for varying angular orientations of a human subject while maintaining a fixed separation distance between the transmitter (TX) and receiver (RX). Although these studies have made important contributions to our understanding of the $\mathrm{mm}$ wave on- and off-body communications channels, they have only considered scenarios where the person has been stationary or made dynamic movements at a particular location.

Against this background, the contributions of this letter may now be highlighted as follows. For the first time, we investigate the first-order statistical characteristics of off-body communication channels operating at $60 \mathrm{GHz}$ when a hypothetical user walks toward and away from a base station. These truly mobile measurements allow us to simultaneously record the path loss, large-scale, and small-scale fading to provide a detailed description of the channel. Furthermore, we perform our measurements over a diverse range of operating environments (both indoor and outdoor) to ensure the generality and applicability of our analysis. Finally, we employ well-known fading models to characterize the signal fluctuation in line-of-sight (LOS) and non-LOS (NLOS) conditions that allow the channels presented here to be readily reproduced.

\section{Measurement System AND EXPERIMENTS}

The experiments conducted in this study were all carried out in the European $(59-66 \mathrm{GHz})$ unlicensed Industrial, Scientific and Medical band at an operating frequency of $60 \mathrm{GHz}$. The measurement system consisted of a Hittite HMC6000LP711E mm-wave TX module and Hittite HMC6001LP711E mm-wave RX module, both containing on-chip, low-profile antennas. The in-package antennas offer $+7.5 \mathrm{dBi}$ gain with a maximum 


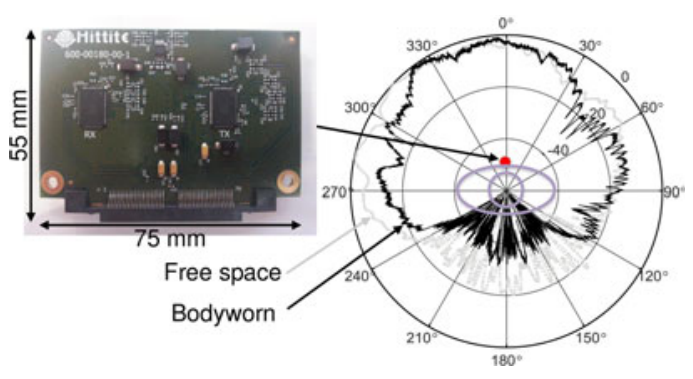

Fig. 1. Measured free-space and bodyworn azimuthal radiation patterns when the TX was positioned at the front-central chest region.

equivalent isotropically radiated power (EIRP) of $+21.1 \mathrm{dBm} .{ }^{1}$ The TX unit was configured to deliver a continuous wave signal operating at $60.1 \mathrm{GHz}$ using the maximum EIRP. The $60-\mathrm{GHz}$ TX was then positioned on the front-central chest region of an adult male of height $1.83 \mathrm{~m}$ and mass $78 \mathrm{~kg}$ so that the antenna was parallel to the body surface. The TX was secured to a harness worn by the test subject using a small strip of Velcro (approximately $6 \mathrm{~mm}$ from the body surface). The measured free-space and bodyworn azimuthal radiation patterns are given in Fig. 1. As shown in Fig. 1, due to the interaction between the human body and antenna, the antenna radiation pattern was slightly distorted when the unit was placed on the human body, but interestingly, the forward gain was not significantly affected. The complex baseband output of the RX module was connected to port 1 of a Rohde \& Schwarz ZVB-8 vector network analyzer (VNA) using an I/Q differential splitter network and subminiature version A barrel connectors. The setup was configured to collect samples of the $b_{1}$ wave quantity with a bandwidth of $100 \mathrm{kHz}$ and a sample rate of $118 \mathrm{~Hz}$. The $60-\mathrm{GHz} \mathrm{RX}$, which acted as a hypothetical mm-wave base station, was positioned on a polyvinyl chloride stand at a height of $2.38 \mathrm{~m}$ from the floor level such that the antenna was vertically polarized for all measurements.

Four different measurement environments were considered, namely a hallway (floor area: $17.38 \times 1.40 \mathrm{~m}^{2}$ ), an open office area (floor area: $10.62 \times 12.23 \mathrm{~m}^{2}$ ), an anechoic chamber (floor area: $5.40 \times 10.00 \mathrm{~m}^{2}$ ), and an outdoor car park as shown in Fig. 2. The indoor hallway and open office measurements were conducted on the first floor of the Institute of Electronics, Communications and Information Technology (ECIT) at Queen's University Belfast, U.K. The indoor environments featured metal-studded dry walls with a metal tiled floor covered with polypropylene-fiber, rubber-backed carpet tiles, and metal ceiling with mineral fiber tiles and recessed louvered luminaries suspended $2.70 \mathrm{~m}$ above the floor level. The open office area contained a number of soft partitions, cabinets, PCs, chairs and desks. The anechoic chamber measurements were conducted in the structure described in [7]. The outdoor measurements were performed in an open car park adjacent to the ECIT building. It should be noted that all of the environments were unoccupied for the duration of the measurements to facilitate pedestrian free off-body channel measurements.

In this study, we considered two different channel conditions, namely 1) mobile LOS, and 2) mobile NLOS, where the test subject walked toward and away from the hypothetical base station in a straight line, respectively. Due to the dissimilar

\footnotetext{
${ }^{1}$ The EIRP was measured in a non-reverberant setting at a separation distance of $0.24 \mathrm{~m}$ using a Keysight E8361C Network Analyzer and a 20-dB gain horn antenna manufactured by Flann (model no. 25240-25).
}
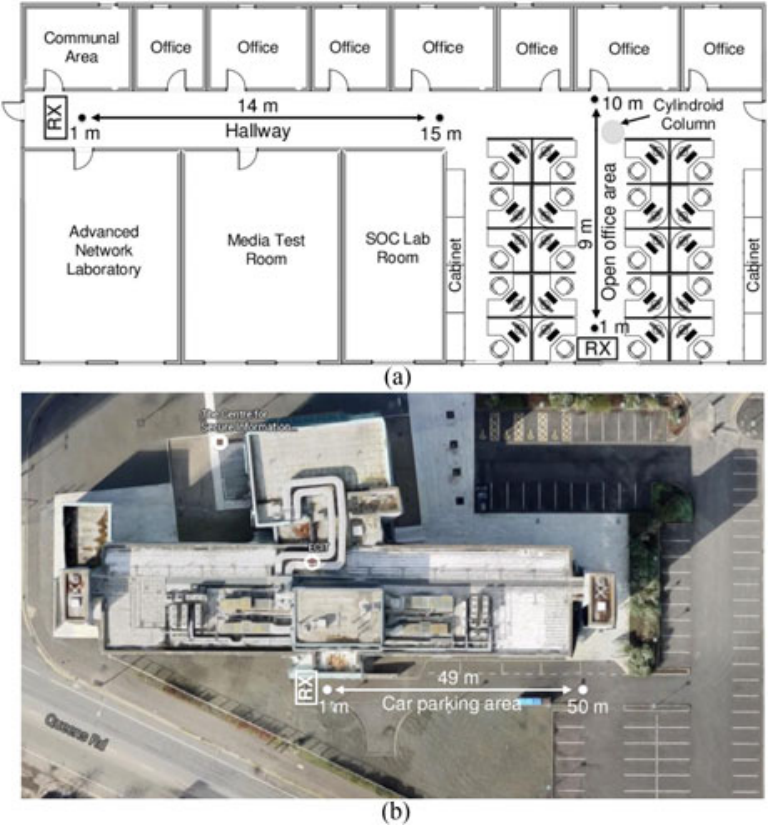

Fig. 2. (a) Indoor hallway $\left(24.33 \mathrm{~m}^{2}\right)$ and open office $\left(129.88 \mathrm{~m}^{2}\right)$ environments; (b) outdoor car park environment (image courtesy of Google Maps).

sizes of each environment, the considered walking distances were different. These were hallway $(14 \mathrm{~m})$, open office $(9 \mathrm{~m})$, anechoic chamber $(7 \mathrm{~m})$, and car park $(49 \mathrm{~m})$. It should be noted that only first $5 \mathrm{~m}$ of the NLOS measurement data in the car park was used in our data analysis due to the received signal power regularly extending below the noise threshold of the receiver beyond this point. The difference between the corresponding lowest recorded signal power and the average noise threshold for each environment are given in Table I. The minimum dataset sizes utilized for our analysis consisted of 1982,1365, 1020, and 474 samples for the hallway, open office, anechoic chamber, and car park environments, respectively. The average walking speed maintained by the test subject throughout all of the experiments was approximately $0.8 \mathrm{~m} / \mathrm{s}$.

\section{DATA ANALYSIS}

The path loss, large-scale, and small-scale fading are mainly responsible for shaping the characteristics of the received signal in wireless communications channels. The path loss is a measure of the signal attenuation between the TX and RX as a function of the separation distance and can be expressed as [8]

$$
P[\mathrm{~dB}]=P_{0}+10 n \log _{10}\left(d / d_{0}\right)
$$

where $P_{0}$ represents the path loss at the reference distance $d_{0}, n$ is the path loss exponent that indicates the rate at which the path loss increases with distance, and $d$ is the TX-RX separation distance. To obtain estimates for $P_{0}$ and $n$, we first removed the EIRP and gain at the RX from the raw signal power received by the VNA and then performed linear regression available in MATLAB. To this end, the elapsed time was first converted into a distance based upon an estimate of the test subject's velocity, i.e., time was mapped into distance. The reference distance, which should be in far-field region of the antenna, was $1 \mathrm{~m}$ for all environments.

The gamma distribution has been widely used to describe large-scale fading because of its excellent fit to empirical data 
TABLE I

Difference Between Lowest Recorded Signal Power and Noise Threshold $\left(D_{S N}\right)$, Path Loss Parameters and Body Shadowing Factors Along With the PARAMETER Estimates FOR the Gamma, Rice, AND NAKAGAMI- $m$ Distributions FOR the LOS AND NLOS CASES

\begin{tabular}{|c|c|c|c|c|c|c|c|c|c|c|c|c|c|c|c|}
\hline \multirow[t]{3}{*}{ Environments } & \multirow{3}{*}{$\begin{array}{r}D_{S N} \\
(\mathrm{~dB})\end{array}$} & \multicolumn{4}{|c|}{ Path loss } & \multirow{3}{*}{$\begin{array}{l}\text { BSF } \\
(\mathrm{dB})\end{array}$} & \multicolumn{4}{|c|}{ Large-scale fading } & \multicolumn{5}{|c|}{ Small-scale fading } \\
\hline & & \multicolumn{2}{|c|}{ LOS } & \multicolumn{2}{|c|}{ NLOS } & & \multicolumn{2}{|c|}{ LOS } & \multicolumn{2}{|c|}{ NLOS } & \multicolumn{3}{|c|}{ LOS } & \multicolumn{2}{|c|}{ NLOS } \\
\hline & & $n$ & $P_{0}(\mathrm{~dB})$ & $n$ & $P_{0}(\mathrm{~dB})$ & & $\alpha$ & $\beta$ & $\alpha$ & $\beta$ & $s$ & $\sigma$ & $K(\mathrm{~dB})$ & $m$ & $\Omega$ \\
\hline Hallway & 12.7 & 1.13 & 42.3 & 1.18 & 58.5 & 16.2 & 7.49 & 0.15 & 7.06 & 0.16 & 0.94 & 0.30 & 6.7 & 2.67 & 1.07 \\
\hline Office & 20.0 & 1.18 & 45.1 & 1.07 & 57.4 & 12.3 & 7.01 & 0.15 & 5.77 & 0.20 & 0.93 & 0.34 & 5.7 & 2.35 & 1.10 \\
\hline Anechoic & 2.2 & 1.41 & 43.0 & 1.86 & 75.5 & 32.5 & 17.39 & 0.06 & 10.27 & 0.11 & 0.98 & 0.16 & 12.5 & 1.94 & 1.12 \\
\hline Car park & 2.6 & 1.53 & 48.7 & 1.98 & 88.8 & 40.1 & 10.30 & 0.11 & 5.11 & 0.23 & 0.97 & 0.17 & 11.9 & 2.74 & 1.10 \\
\hline
\end{tabular}

and its mathematical tractability [9]. Letting $Z$ represent a gamma random variable that is used to model the large-scale fading, the corresponding cumulative distribution function (CDF) can be expressed with the shape parameter $\alpha$ and scale parameter $\beta$ as follows:

$$
F_{Z}(z)=\frac{1}{\Gamma(\alpha)} \gamma\left(\alpha, \frac{z}{\beta}\right)
$$

where $\Gamma(\cdot)$ is the gamma function and $\gamma(\cdot, \cdot)$ is the lower incomplete gamma function.

The Rice and Nakagami- $m$ fading models have gained widespread use to describe small-scale fading for the LOS and NLOS channel conditions, respectively [10]. The CDF of the signal envelope, $R$, in a Rice fading channel can be expressed as follows:

$$
F_{R}(r)=1-Q_{1}\left(\frac{s}{\sigma}, \frac{r}{\sigma}\right)
$$

where $Q_{1}(\cdot)$ denotes the Marcum $Q$-function and $s$ and $\sigma$ are the noncentrality and scaling parameters, respectively. From the parameters $s$ and $\sigma$, we can obtain well-known $K$ factor that is defined as the ratio between the power in the dominant component $\left(s^{2}\right)$ and the power in the scattered component $\left(2 \sigma^{2}\right)$.

In a Nakagami- $m$ fading channel, the CDF of the signal envelope, $R$, can be expressed as

$$
F_{R}(r)=\frac{\gamma\left(m, m r^{2} / \Omega\right)}{\Gamma(m)}
$$

where $m$ is the fading severity parameter and $\Omega=E\left[r^{2}\right]$ is the mean power with $E[\cdot]$ denoting the expectation operator.

\section{RESULTS}

\section{A. Path Loss}

The parameter estimates for $P_{0}$ and $n$ over all of the considered environments are given in Table I along with the body shadowing factor (BSF) that is defined as the difference between $P_{0}$ for the LOS and NLOS scenarios. As an example, Fig. 3(a) and (b) shows the path loss model fits for the LOS and NLOS in the hallway environment, respectively. As anticipated, for all of the environments, $P_{0}$ for the NLOS case was greater than that for the LOS due to the shadowing effects caused by the test subject's body. When comparing the corresponding BSF for all environments, the body shadowing effects were more predominant in the anechoic chamber and car park environments than those observed in the hallway and open office environments. For both the LOS and NLOS scenarios, the estimated path loss exponents for the anechoic chamber and car park environments were greater than those for the hallway and open office environments. This was presumably due to the additional multipath

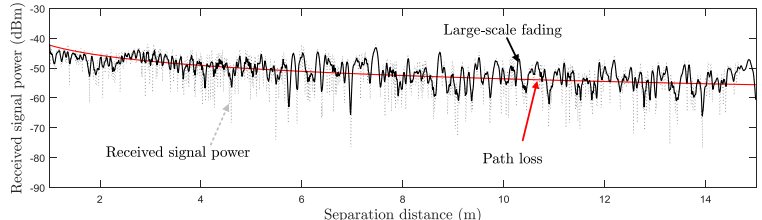

(a)

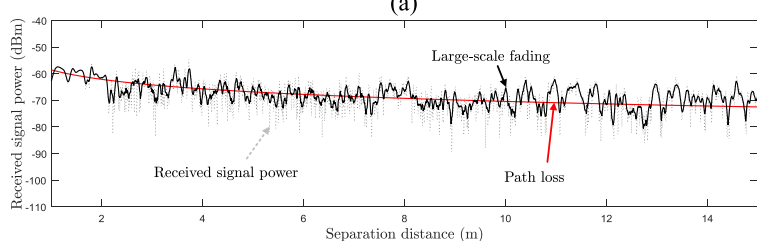

(b)

Fig. 3. Received signal power with the superimposed path loss fit and largescale fading for the (a) LOS and (b) NLOS in the hallway environment.

present in the latter environments, which may have helped to mitigate the shadowing introduced by the body for the NLOS scenario.

\section{B. Large-Scale Fading}

To abstract the large-scale fading from the received signal power, the estimated path loss was first removed from the measurement data using the path loss parameters given in Table I. Then, the local mean was calculated by averaging over a distance of ten wavelengths (or equivalently seven samples). The parameter estimates for the gamma fading model were obtained using maximum likelihood estimation (MLE) performed in MATLAB. Fig. 3(a) and (b) shows the large-scale fading overlaid on the received signal power for the LOS and NLOS in the hallway environment, respectively. As an example of the model fitting obtained for the large-scale fading, Fig. 4 shows the CDFs of the gamma distribution fitted to the empirical data for the LOS and NLOS walking scenarios in each environment. It is clear that this probability model provides an adequate agreement with the measured data for all of the considered cases. ${ }^{2}$ To allow the reader to reproduce their own simulated large-scale fading data based on the empirical data reported here, Table I provides the parameter estimates for the gamma distribution over all four environments. Reviewing Table I, it is clear that the $\alpha$ parameter for the LOS large-scale data was greater than that for the NLOS, suggesting that the off-body channel for the LOS walking scenario was less susceptible to variations caused by large-scale fading compared to the NLOS case.

\footnotetext{
${ }^{2} \mathrm{~A}$ Kolmogorov-Smirnov (K-S) test was performed at the $1 \%$ significance level for all large-scale fades above the 0.01 cumulative probability level. It failed to reject the null hypothesis that the data followed the specified distribution for $50 \%$ and $75 \%$ of all LOS and NLOS cases, respectively.
} 


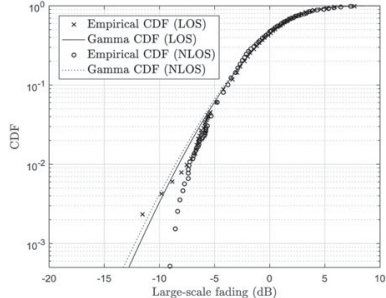

(a)

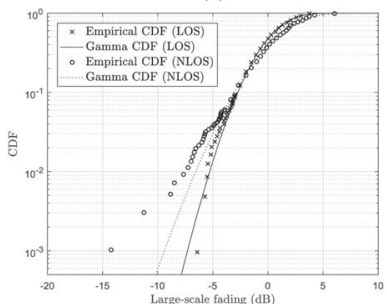

(c)

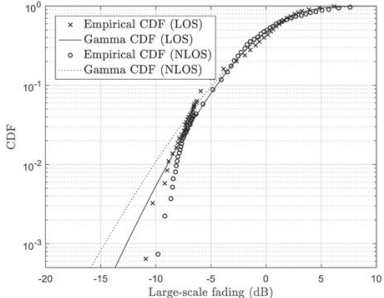

(b)

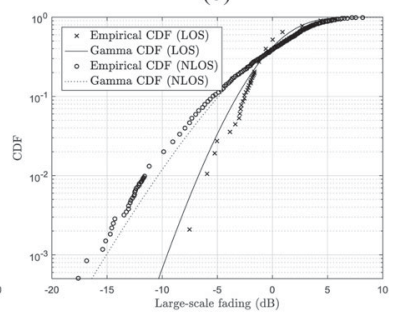

(d)
Fig. 4. Empirical CDFs of the large-scale fading with corresponding gamma CDF fit: (a) Hallway, (b) Open office, (c) Anechoic chamber, and (d) Car park.

\section{Small-Scale Fading}

The small-scale fading was extracted by removing both the path loss and large-scale fading from the measurement data as detailed above before transforming the data to its linear amplitude. Similar to the large-scale fading, the parameter estimates for the Rice and Nakagami- $m$ fading models were obtained using MLE. Table I shows the parameter estimates for the Rice and Nakagami- $m$ fading models for the LOS and NLOS walking scenarios, respectively. As expected, the $K$ factor for the LOS case was greater than unity for all of the considered environments indicating that a strong dominant signal component existed. When comparing the $K$ factors across all four environments, the $K$ factors for the anechoic chamber and car park environments were larger than those for the hallway and open office environments. This was most likely due to the varying levels of scattered signals found in each environment. As shown in Table I, the scattered signal power $\left(2 \sigma^{2}\right)$ for the hallway and open office environments was greater than the anechoic chamber and outdoor car park environments, while the dominant signal power $\left(s^{2}\right)$ was almost the same for all of the environments. As shown in Table I, the $m$ parameter estimates were greater than unity for all of the environments. This indicates that the fluctuations of the signal envelope observed in NLOS scenarios were less severe than those experienced in Rayleigh fading channels $(m=1)$.

As an example of the model fitting for the small-scale fading, Fig. 5 shows the excellent fits of the Rice and Nakagami- $m$ fading models to the measurement data for the LOS and NLOS cases, respectively. ${ }^{3}$ Interestingly, the shape of the CDFs for the anechoic chamber and car park environments (less multipath) were significantly different between the LOS and NLOS orientations. Specifically, the shape of the CDF for the LOS case was much more constricted (in fact, the small-scale fading becomes increasingly deterministic) than that observed for the NLOS condition. This suggests that LOS channels in sparse environments will not suffer from significant fluctuations due to small-scale fading.

\footnotetext{
${ }^{3}$ Similarly, the K-S test performed at the $1 \%$ significance level for all smallscale fades above the 0.01 cumulative probability level failed to reject the null hypothesis for 50\% and $75 \%$ of all LOS and NLOS cases, respectively.
}

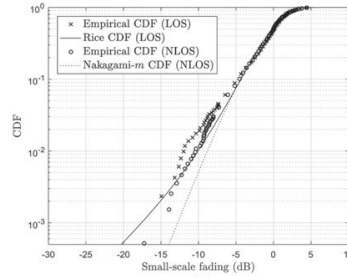

(a)

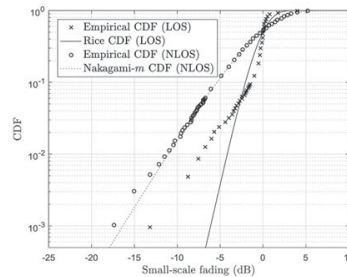

(c)

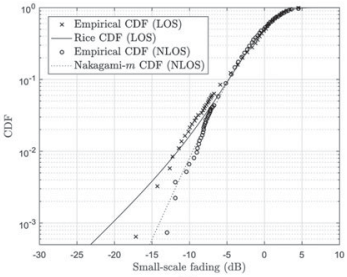

(b)

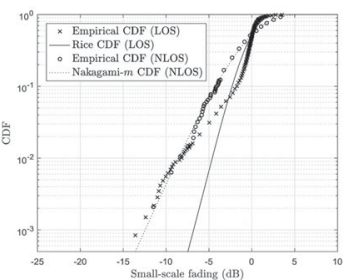

Fig. 5. Empirical CDFs of the small-scale fading with the corresponding Rice and Nakagami- $m$ CDF fits: (a) Hallway, (b) Open office, (c) Anechoic chamber, and (d) Car park.

\section{CONCLUSION}

The first-order characteristics of dynamic off-body communications channels at $60 \mathrm{GHz}$ within indoor and outdoor environments have been investigated in terms of path loss, large-scale, and small-scale fading when the bodyworn node was positioned on the front-central chest region. From the estimated path loss at the reference distance, it was found that the impact of body shadowing on the off-body link was more pronounced in the anechoic chamber and car park environments, where there existed less opportunities for multipath signal propagation compared to the hallway and open office environments. Over all of the measurement scenarios considered in this study, the gamma distribution was found to well describe the large-scale fading. Furthermore, the Rice and Nakagami- $m$ distributions provided a good fit to small-scale fading observed in the LOS and NLOS off-body links, respectively.

\section{REFERENCES}

[1] K. Venugopal, M. C. Valenti, and R. W. Heath, Jr., "Analysis of millimeter wave networked wearables in crowded environments," in Proc. Asilomar Conf. Signals, Syst., Comput., Nov. 2015, pp. 872-876.

[2] N. Chahat, G. Valerio, M. Zhadobov, and R. Sauleau, "On-body propagation at $60 \mathrm{GHz}$," IEEE Trans. Antennas Propag., vol. 61, no. 4, pp. 18761888, Apr. 2013.

[3] L. Petrillo, T. Mavridis, J. Sarrazin, A. Benlarbi-Delai, and P. De Doncker, "Statistical on-body measurement results at $60 \mathrm{GHz}$," IEEE Trans. Antennas Propag., vol. 63, no. 1, pp. 400-403, Jan. 2015.

[4] S. L. Cotton, W. G. Scanlon, and B. K. Madahar, "Millimeter-wave soldierto-soldier communications for covert battlefield operations," IEEE Commun. Mag., vol. 47, no. 10, pp. 72-81, Oct. 2009.

[5] S. L. Cotton, W. G. Scanlon, and P. S. Hall, "A simulated study of cochannel inter-BAN interference at $2.45 \mathrm{GHz}$ and $60 \mathrm{GHz}$," in Proc. Eur. Wireless Technol. Conf., Sep. 2010, pp. 61-64.

[6] T. Mavridis, L. Petrillo, J. Sarrazin, D. Lautru, A. Benlarbi-Delai, and P. De Doncker, "Theoretical and experimental investigation of a $60-\mathrm{GHz}$ off-body propagation model," IEEE Trans. Antennas Propag., vol. 62, no. 1, pp. 393-402, Jan. 2014.

[7] S. L. Cotton and W. G. Scanlon, "Characterization and modeling of the indoor radio channel at $868 \mathrm{MHz}$ for a mobile bodyworn wireless personal area network," IEEE Antennas Wireless Propag. Lett., vol. 6, pp. 51-55, 2007.

[8] T. S. Rappaport, Wireless Communications: Principle and Practice. Englewood Cliffs, NJ, USA: Prentice-Hall, 2002.

[9] A. Abdi and M. Kaveh, "On the utility of gamma PDF in modeling shadow fading (slow fading)," in Proc. Veh. Technol. Conf., May 1999, vol. 3, pp. 2308-2312.

[10] M. K. Simon and M.-S. Alouini, Digital Communication Over Fading Channels. New York, NY, USA: Wiley, 2005. 